\title{
Lignes directrices pour l'expertise médicale des maladies rhumatismales et des séquelles rhumatismales d'accident
}

Société suisse de rhumatologie, groupe de travail "médecine d'assurance»

\section{Rôle des lignes directrices}

\subsection{Définition du concept}

- Les lignes directrices sont des recommandations sur la manière de procéder à une expertise. Elles fixent des standards notamment de contenu, de forme et de langage, en matière de processus et d'exigences posées aux experts.

- Elles sont conçues pour renforcer l'unité de doctrine parmi les rhumatologues, et donc améliorer la qualité et la rentabilité.

- Le respect des lignes directrices est une garantie de sécurité, tout écart doit être justifié.

\subsection{Destinataires}

Ces lignes directrices s'adressent aux rhumatologues qui réalisent des expertises.

\section{Principes}

\subsection{Signification d'une expertise}

- Les expertises médicales sont avant tout des moyens de preuve.

- La réalisation d'une expertise est généralement demandée lorsque l'état médical d'une personne n'est pas clair pour l'instance chargée d'appliquer le droit (administration ou tribunal en cas de litige).

- L'expertise aide cette instance à décider si l'assuré est en droit de bénéficier de prestations d'assurance, et le cas échéant, de quelles prestations.

- Le médecin qui procède à l'expertise a ici une fonction d'expert*. Néanmoins, la responsabilité de l'attribution ou du refus des prestations d'assurance incombe toujours à l'instance chargée d'appliquer le droit.

- Le rapport d'expertise doit être rédigé dans un langage aisément compréhensible par l'instance chargée d'appliquer le droit.

Pour une meilleure lisibilité du texte, seule la forme masculine est employée, mais nous nous adressons bien entendu aux collègues des deux sexes.

\subsection{Rôle de l'expert}

- La réalisation d'une expertise implique pour le médecin un changement de perspective: tandis que dans le cadre thérapeutique, les de- mandes du patient sont au premier plan, dans la situation d'expertise, il s'agit prioritairement de mettre en évidence une situation de manière objective.

- L'expert est tenu d'observer la plus grande neutralité et la plus grande objectivité possible. La prise de décision ne doit être influencée ni par les intérêts du mandant, ni par ceux de l'assuré. Pour ces raisons, un rhumatologue traitant ne doit pas réaliser d'expertise sur ses propres patients.

- En se fondant sur l'état des connaissances de la science médicale, l'expert doit indiquer de manière probante à l'instance chargée d'appliquer le droit si l'on est en présence ou pas d'une maladie particulièrement grave et d'un pronostic défavorable (sur la notion d'«atteinte durable à la santé», voir le paragraphe 2.6)

\subsection{Critères juridiques à remplir par l'expertise}

Le Tribunal fédéral des assurances (TFA) a formulé de la manière suivante les critères juridiques que doit remplir toute expertise médicale réalisée pour l'assurance sociale (ATF 125 V 351):

Pour la valeur probante d'un rapport d'expertise, il importe:

- que les points litigieux aient fait l'objet d'une étude fouillée,

- que le rapport se fonde sur des examens complets,

- qu'il prenne en considération les plaintes exprimées,

- qu'il ait été établi en pleine connaissance du dossier (anamnèse),

- que la description du contexte médical et l'analyse de la situation médicale soient claires, et

- que les conclusions de l'expert soient bien motivées.

\subsection{Exigences posées à l'expert}

- La condition préalable est que l'expert ait une formation de spécialiste en rhumatologie. Il doit en outre avoir une excellente connaissance de l'état actuel de la science médicale (médecine fondée sur les preuves).

- De plus, l'expert doit acquérir des connaissances fondamentales en médecine des assu- 
rances, en fréquentant par exemple les cours pour experts de Swiss Insurance Medicine (www.swiss-insurance-medicine.ch) ou en obtenant le Certificat de capacité de médecinconseil.

- Enfin, il ne faut pas sous-estimer l'importance de l'expérience personnelle, qui justifie sa qualité d'expert.

\subsection{Juristes et médecins:}

\section{deux modes de pensée différents}

- Le médecin doit pouvoir pénétrer l'univers réflexif du juriste et le comprendre. Le juriste pense de manière normative (déductive), il tire ses conclusions de normes (lois, ordonnances, jurisprudence) qu'il applique aux cas particuliers. A l'inverse, le médecin procède de manière scientifique, c'est-à-dire inductive: il observe des cas particuliers et tente d'en dégager une règle générale (recherche empirique).

- Dans tous les cas, les instances chargées d'appliquer le droit souhaitent obtenir des réponses explicites à leurs questions, même lorsque la situation n'est pas entièrement claire du point de vue médical. Il est de la responsabilité de l'expert de faire tout son possible pour aider l'instance chargée d'appliquer le droit à résoudre les questions posées, mais aussi d'attirer son attention sur les limites de la science médicale.

\subsection{Notion d'«atteinte durable à la santé»}

- En droit des assurances sociales, la notion d'atteinte durable à la santé occupe une place centrale. Pour qu'une invalidité soit reconnue au sens juridique, trois conditions doivent être remplies: 1) une atteinte de longue durée de la santé physique ou mentale (atteinte durable à la santé), 2) une incapacité totale ou partielle d'accomplir un travail, et 3) un lien de cause à effet entre l'atteinte à la santé et l'incapacité d'accomplir un travail [1].

- Le rhumatologue expert évalue l'état de santé et donne son avis sur la question de savoir dans quelle mesure celui-ci entraîne une incapacité de travail.

- Dans l'appréciation globale de l'état de santé, il est essentiel d'indiquer si un concept thérapeutique approprié peut permettre d'obtenir une amélioration sensible ou si le pronostic est défavorable. Un avis sur les thérapies employées jusqu'alors et leur efficacité, sur de nouvelles possibilités thérapeutiques prometteuses, ainsi que sur le pronostic constituent une partie essentielle de toute expertise.
- Dans la jurisprudence actuelle (ATF 127 V 294), les troubles de la santé essentiellement imputables aux conditions sociales ne relèvent pas de l'assurance-invalidité ni des autres assurances sociales pouvant prendre en charge les incapacités de travail ayant des causes médicales (assurance accidents, assurance militaire, prévoyance professionnelle). Sur la question de l'invalidité, la jurisprudence suisse s'en tient à une conception biopsychique de la maladie [1], contrairement à la médecine, qui travaille depuis longtemps avec un modèle bio-psycho-social. Cela permet de délimiter le champ des prestations de l'assurance-invalidité par rapport à celui de l'aide sociale et de l'assurance-chômage. Dans ce contexte, l'assurance-invalidité demande à l'expert de préciser le rôle joué par les facteurs dits «étrangers à l'invalidité» (exemples: une situation personnelle, familiale ou sociale difficile, un manque de connaissances professionnelles ou linguistiques). Il peut ici arriver que l'expert ait des difficultés à tracer la frontière entre les facteurs sociaux et non sociaux qui jouent un rôle dans la maladie. Là encore, l'une des missions fondamentales de l'expert consiste à attirer l'attention sur les limites de ses possibilités.

\subsection{Place de l'expertise rhumatologique (expertise partielle) dans le cadre de} I'expertise bidisciplinaire et pluridisciplinaire

- Le rhumatologue qui participe à une expertise pluridisciplinaire doit veiller à rester dans son domaine de spécialité et à ne pas empiéter sur une spécialité dans laquelle il n'est pas compétent.

- Le rhumatologue fera notamment preuve de la plus grande prudence dans l'usage des diagnostics d'amplification, de simulation ou de somatisation. Il s'efforcera de décrire le comportement du malade sans porter de jugement de valeur. Une description du type «syndrome hémialgique à droite sans corrélat somatique adéquat» laisse au psychiatre toute latitude pour apprécier si l'on est ou pas en présence d'un trouble psychique à caractère de maladie.

\section{Modalités pratiques}

\subsection{Etude du dossier et information}

\section{Etude du dossier}

Il incombe en principe au mandant de l'expertise ainsi qu'à l'assuré (dans le cadre de son devoir de collaborer) de mettre à disposition de 
l'expert l'intégralité des documents médicaux (dossiers, examens d'imagerie, analyses de laboratoire, etc). Les documents présentés doivent être étudiés et appréciés de manière approfondie. Les documents pertinents doivent être résumés dans le rapport d'expertise, avec indication de leur auteur et de leur date de réalisation.

\section{Communication}

Le recours éventuel à un traducteur est à la charge du mandant de l'expertise.

\section{Information}

L'identité du mandant, la levée du secret médical, la nature de la mission (questions posées), la position/neutralité de l'expert, l'importance ainsi que les tenants et aboutissants de l'expertise doivent être communiqués à l'assuré.

Résultats de l'examen/éclaircissements Ils sont précisés et appréciés dans le rapport.

\section{Copies}

Le rapport écrit est communiqué exclusivement au mandant de l'expertise. L'assuré a le droit (droit du patient) de demander à ce dernier une copie du rapport, dont il peut disposer à sa guise.

\subsection{Interrogation du patient}

L'interrogation approfondie de l'assuré doit fournir des renseignements sur:

\section{Evolution de la maladie}

Début, antécédents familiaux, répercussions/ influences de maladies/d'accidents antérieurs, effets des positions et des sollicitations, résultats d'examens effectués jusqu'alors, interventions et thérapies. Il peut être nécessaire de récolter des renseignements auprès d'autres sources.

\section{Accidents}

Etat antérieur, déroulement de l'accident, symptômes initiaux, évolution, thérapies et réactions aux thérapies.

\section{Situation actuelle}

Symptômes, douleurs, limitations dans les activités et la vie sociale.

\section{Déroulement d'une journée}

La reconstitution minutieuse d'une journée fournit des indications précieuses sur les répercussions de la maladie sur les différents domaines de la vie quotidienne.

\section{Activités/engagements}

Activités et obligations extraprofessionnelles, hobbies.

\section{Intégration}

Familiale, professionnelle, sociale.

\section{Initiatives et compliance du patient}

Thérapies, entraînement, tentatives de travailler, mesures pour contribuer à l'atténuation des troubles.

\section{Evaluation de la situation par le patient}

Estimation par l'assuré de sa propre capacité de travail, solutions, attitude face à l'avenir, problèmes avec l'assurance, requêtes formulées auprès de l'assurance.

\subsection{Examens complémentaires}

Les examens de laboratoire

Les examens de laboratoire ne sont indiqués que s'ils sont d'une importance décisive pour l'argumentation ou pour répondre définitivement aux questions posées, notamment s'ils permettent d'établir avec certitude ou d'exclure un diagnostic. L'expert doit être en mesure de justifier ces examens (connaissance de la probabilité prétest, sensibilité, spécificité, valeur prédictive). Les examens servant au suivi médical du patient sont intégrés dans les recommandations.

\section{Imagerie médicale}

La radiographie classique constitue l'examen standard. En règle générale, les examens des zones qui posent problème doivent être refaits dès lors que les images dont on dispose datent de plus de six mois. Lorsque les douleurs n'évoluent pas et que l'examen clinique n'indique aucune modification (par rapport aux éléments du dossier), des radiographies plus anciennes peuvent suffire. Des examens complémentaires ne seront réalisés que pour éliminer tout doute quant au diagnostic.

\section{Evaluation du poste de travail}

Une évaluation du poste de travail n'entre en ligne de compte que si l'assuré est toujours en poste. L'employé et l'employeur doivent tous deux donner leur consentement (oral) à cet examen. Il est en général complété par une exploration visant à procéder à des adaptations ou à des modifications (ergonomiques) du profil de l'assuré.

\section{Situation sociale et professionnelle Charges, conditions, obligations.}




\section{Evaluation de la capacité fonctionnelle (ECF)}

Si une ECF est envisagée, le rapport d'expertise doit indiquer pourquoi elle a été effectuée ou pourquoi on y a renoncé.

\section{Indications pertinentes pour la réalisation} d'une ECF

- Evaluation des capacités au niveau général ou local (par exemple ceinture scapulaire), en fonction de la sollicitation physique ou de la position;

- évaluation de la motivation du patient à réaliser certains efforts;

- évaluation de sa constance à l'effort;

- justification complémentaire: évaluation du potentiel de réadaptation.

\section{Mauvaises indications pour la réalisation}

d'une ECF

- Auto-limitation manifeste;

- manque manifeste de motivation (pour les changements);

- amplification manifeste (des douleurs et/ou des difficultés fonctionnelles);

- simulation manifeste.

\subsection{Rédaction du rapport d'expertise}

\section{Etat du dossier}

Plus les documents antérieurs sont nombreux, plus il est important qu'ils soient listés dans l'ordre chronologique et résumés avec concision, de manière à ce que l'essentiel en soit dégagé. Tous les documents utilisés dans le cadre d'une justification ultérieure doivent être présentés dans cette section. Une simple liste ne suffit pas si elle n'est pas accompagnée d'un résumé.

\section{Anamnèse}

L'anamnèse comprend l'anamnèse personnelle, médicale, professionnelle et sociale, ainsi que la description des douleurs actuelles. Il ne suffit cependant pas de décrire les symptômes, il s'agit aussi d'évoquer leurs répercussions sur l'activité et la participation du patient. Les douleurs doivent être décrites en détail, si possible en utilisant les propres mots de l'assuré. Dans cette section, les descriptions sommaires, exprimées en langage médical (par exemple «cervicobrachialgie permanente côté droit») doivent être évitées.

\section{Constatations}

L'état du patient doit être décrit de manière détaillée et avec toute la précision qui s'impose, en particulier les éléments pathologiques mis en évidence par l'examen. Ces derniers doivent également inclure les limitations fonctionnelles physiques qui sont à l'origine d'éventuels déficits somatiques dans les activités et la vie sociale. Dans cette section, il convient par ailleurs de décrire les observations que l'expert peut faire lors de l'interrogation du patient et lors de l'examen clinique, ainsi que tout ce qui peut renseigner sur le comportement développé par le patient par rapport à sa maladie (illness behaviour). Dans le rapport d'expertise, les abréviations qui figurent habituellement dans les rapports médicaux doivent être explicitées lorsqu'elles sont mentionnées pour la première fois, étant donné que les destinataires ne sont en général pas des spécialistes en médecine.

\section{Les diagnostics}

Les diagnostics doivent être énumérés dans une section à part et classés par ordre d'importance. Il importe d'opérer une distinction claire entre les constatations et les diagnostics. Il peut être utile de classer les diagnostics en deux catégories, selon qu'ils restreignent la capacité de travail ou non. L'expert ne doit poser que des diagnostics qui touchent son domaine de spécialité, et respecter la terminologie scientifique propre à sa spécialité [2]. Les suppositions de diagnostic sont à présenter comme telles, l'expression «état post...», ou «état après» doit être évitée (et remplacée par l'indication d'un intervalle de temps ou d'une date). Les diagnostics qui ne relèvent pas de la spécialité de l'expert doivent pouvoir être justifiés sur la base d'éléments du dossier. Le diagnostic différentiel n'a pas sa place dans la liste des diagnostics. Lorsqu'un diagnostic doit rester ouvert, cela doit être expressément déclaré et explicité dans la section «Evaluation».

\section{Evaluation}

L'évaluation constitue le cœur de l'expertise, dans lequel l'évolution de la maladie, les diagnostics et les réflexions qui ont guidé le diagnostic différentiel sont expliqués et pondérés. A ce stade, il est important que l'expert établisse un lien entre l'atteinte à la santé (trouble fonctionnel) constatée et ses répercussions sur l'activité et la participation du patient. Les fonctions résiduelles (ressources) doivent être décrites et évaluées au même titre que les déficits. En cas de difficulté à établir la situation de façon certaine, le degré de probabilité doit être indiqué.

\section{Catalogue de questions}

Les réponses aux questions doivent être claires et mentionner le cas échéant le degré de probabilité. L'expert ne doit pas succomber à la tentation de répondre à des questions auxquelles il ne peut 
répondre de façon probante. Dans cette hypothèse, il faut un commentaire approprié. L'expert ne doit prendre position que sur des questions relatives à son propre domaine de spécialité et de compétence.

\section{Evaluation de la capacité de travail}

L'évaluation de la capacité fonctionnelle physique et de la capacité productive du patient doit être décrite et justifiée avec la précision qui convient. L'expert doit également prendre position sur d'éventuelles évaluations effectuées par rapport au poste de travail ou au domicile (ménage) du patient. Lorsque la reprise de l'activité professionnelle antérieure ne peut plus être envisagée, l'expert doit décrire le profil de compétences d'une activité de substitution. La restriction de la productivité au travail peut prendre la forme d'une réduction de la charge et/ou du temps de travail. Il faut toujours commencer par examiner si une baisse de la charge de travail peut se faire sur la base d'une présence à plein temps. C'est seulement lorsqu'on ne saurait attendre du patient qu'il soit présent à plein temps que l'on peut envisager une réduction du temps de travail.

\section{Mesures médicales}

Ce qui intéresse particulièrement le juriste chargé d'appliquer le droit c'est de savoir si la thérapie suivie jusqu'alors était adéquate, si l'assuré s'est montré coopératif (compliance) et s'il existe des traitements exigibles susceptibles d'améliorer la capacité de travail et le pronostic. Lorsque le patient présente des séquelles d'accidents, il importe souvent aussi de savoir si un traitement donné permettra d'atténuer encore substantiellement les troubles ou seulement de les maintenir à leur niveau actuel.

\section{Mesures professionnelles}

Lorsqu'il formule des recommandations sur d'éventuelles mesures professionnelles à prendre, l'expert doit se fonder sur les handicaps (déficits) et les fonctions résiduelles (ressources) constatés pour décrire le profil de compétences d'une activité de substitution. Il ne doit pas proposer de métiers concrets.

\section{Pronostic}

Concernant la notion d'atteinte durable à la santé, il est important de savoir si un traitement réaliste, convenable et disponible permettrait d'obtenir à brève ou moyenne échéance une amélioration notable de l'état de santé du patient, ou si le pronostic est au contraire défavorable. Dans la mesure où il est souvent impossi- ble de formuler un pronostic à long terme, il convient de se limiter à peu près aux deux années à venir.

\section{Glossaire}

\section{Accident (sens juridique)}

Est réputé accident toute atteinte dommageable, soudaine et involontaire, portée au corps humain par une cause extérieure extraordinaire qui compromet la santé physique, mentale ou psychique ou qui entraîne la mort (art. 4 LPGA).

\section{Aggravation}

- permanente: suite à un accident, la maladie initiale peut connaître une aggravation permanente, ce qui signifie que le status quo ante ne sera plus jamais retrouvé et que le patient présentera toujours un état déficitaire;

- accélératrice: la maladie indépendante de l'accident connaît une aggravation accélératrice lorsque l'accident la fait évoluer plus tôt que prévu, en accélère l'évolution ou lui fait atteindre un stade douloureux qui ne s'atténuera plus;

- temporaire: l'état antérieur est temporairement aggravé par l'accident, puis on retrouve le status quo ante [3].

\section{Amplification}

Exagération volontaire d'un trouble existant, présenté comme plus grave ou plus intense qu'il n'est en réalité, dans le but d'en tirer des avantages (matériels).

\section{Amplification des symptômes}

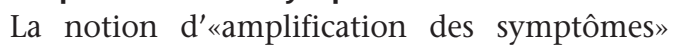
n'est ni un diagnostic, ni un concept nosologique. Elle ne sert qu'à décrire un phénomène dans lequel on retrouve pour l'essentiel les observations suivantes: le patient se plaint de symptômes exagérément intenses, éventuellement étendus du point de vue de leur topographie (contredisant l'expérience clinique que l'on peut avoir de la problématique médicale concernée), il présente une limitation fonctionnelle étendue et une forte restriction de ses activités, ainsi qu'un manque de volonté lorsqu'il s'agit de participer aux évaluations fonctionnelles. On relève des incohérences évidentes lors de l'examen.

\section{ATF}

Arrêts du Tribunal fédéral ou du Tribunal fédéral des assurances publiés dans le Recueil officiel du droit fédéral. Toutes les décisions matérielles peuvent être consultées sur Internet (www. bger.ch). 


\section{Degré de preuve}

Pour être reconnu par l'assurance sociale, un état de fait (par exemple l'imputabilité d'un trouble à un accident) doit être établi au moins avec une vraisemblance prépondérante. Une supposition ou une simple possibilité ne suffisent pas.

- possibilité: vraisemblance inférieure à 50\%;

- vraisemblance prépondérante: (nettement) supérieure à $50 \%$;

- vraisemblance proche de la certitude: presque $100 \%$.

\section{Exigibilité}

Ce terme juridique n'a jusqu'ici été défini dans aucune loi. Dans le langage courant, il désigne le comportement que l'on est en droit d'attendre ou d'exiger d'une personne, même s'il implique pour cette dernière des désagréments ou des sacrifices. D'un point de vue médical, l'exigibilité varie en fonction des capacités et des handicaps que le patient présente par rapport au profil exigé pour un poste de travail donné. La capacité de travail et l'exigibilité sont deux notions très liées. Le médecin considérera en règle générale qu'on ne peut plus parler d'exigibilité lorsqu'un patient souffre d'une maladie lourde assortie de mauvais pronostics et qui l'empêche totalement ou partiellement d'exercer un travail adéquat.

\section{Impotence}

Est réputée impotente toute personne qui, en raison d'une atteinte à sa santé, a besoin de façon permanente de l'aide d'autrui ou d'une surveillance personnelle pour accomplir des actes élémentaires de la vie quotidienne (art 9. LPGA).

\section{Incapacité de gain}

Est réputée incapacité de gain toute diminution de l'ensemble ou d'une partie des possibilités de gain de l'assuré sur un marché du travail équilibré dans son domaine d'activité, si cette diminution résulte d'une atteinte à sa santé physique, mentale ou psychique et qu'elle persiste après les traitements et les mesures de réadaptation exigibles (art. 7 LPGA).

\section{Incapacité de travail}

Est réputée incapacité de travail toute perte, totale ou partielle, de l'aptitude de l'assuré à accomplir dans sa profession ou son domaine d'activité le travail qui peut raisonnablement être exigé de lui, si cette perte résulte d'une atteinte à sa santé physique, mentale ou psychique. En cas d'incapacité de travail de longue durée, l'activité qui peut être exigée de lui peut aussi relever d'une autre profession ou d'un autre domaine d'activité (art. 6 LPGA).

\section{Invalidité}

Est réputée invalidité l'incapacité de gain totale ou partielle qui est présumée permanente ou de longue durée (art. 8, al. 1 LPGA).

\section{LAI}

Loi fédérale sur l'assurance-invalidité (RS 831.20).

\section{LPGA}

Loi fédérale sur la partie générale du droit des assurances sociales (RS 830.1).

\section{Maladie (sens juridique)}

Est réputée maladie toute atteinte à la santé physique, mentale ou psychique qui n'est pas due à un accident et qui exige un examen ou un traitement médical ou provoque une incapacité de travail (art. 3 LPGA).

\section{Mesures médicales}

Sont considérés comme mesures médicales au sens de l'art. 12 LAI notamment les actes chirurgicaux, physiothérapeutiques et psychothérapeutiques qui visent à supprimer ou à atténuer les séquelles d'une infirmité congénitale, d'une maladie ou d'un accident - caractérisées par une diminution de la mobilité du corps, des facultés sensorielles ou des possibilités de contact - pour améliorer de façon durable et importante la capacité de gain ou la capacité d'accomplir des travaux habituels ou préserver cette capacité d'une diminution notable. Les mesures doivent être considérées comme indiquées dans l'état actuel des connaissances médicales et permettre de réadapter l'assuré d'une manière simple et adéquate (art. 2 RAI).

\section{Moyens auxiliaires}

L'assuré a droit, d'après une liste que dressera le Conseil fédéral, aux moyens auxiliaires dont il a besoin pour exercer une activité lucrative ou accomplir ses travaux habituels, pour maintenir ou améliorer sa capacité de gain, pour étudier, apprendre un métier ou se perfectionner, ou à des fins d'accoutumance fonctionnelle. Les frais de prothèses dentaires, de lunettes et de supports plantaires ne sont pris en charge par l'assurance que si ces moyens auxiliaires sont le complément important de mesures médicales de réadaptation (art. $21 \mathrm{LAI}$ ).

\section{Obligation de réduire le dommage}

L'obligation de réduire le dommage pose pour principe que l'on peut attendre de la personne lésée qu'elle prenne les mesures que tout individu raisonnable prendrait dans la même situation s'il ne pouvait prétendre à des prestations 
d'aucune sorte. Comme exemples de démarches, on peut citer la recherche d'un emploi ou le recours à des soins médicaux dont les frais sont remboursés par l'assurance-maladie.

\section{Prestations complémentaires}

Les prestations complémentaires à l'AVS et à l'AI sont versées lorsque les rentes et les autres revenus d'une personne ne suffisent pas à couvrir ses besoins vitaux. Toute personne peut en bénéficier si elle en remplit les conditions d'octroi.

\section{RAI}

Règlement sur l'assurance-invalidité (RS 831.201).

\section{Reclassement}

L'assuré a droit au reclassement dans une nouvelle profession si son invalidité rend cette mesure nécessaire et que sa capacité de gain peut ainsi, selon toute vraisemblance, être maintenue ou améliorée (art. 17 LAI).

RS

Recueil systématique du droit fédéral. Les lois et ordonnances sont disponibles sur Internet, classées par leur numéro RS (www.admin.ch).

\section{Simulation}

Action de feindre volontairement une pathologie à des fins déterminées, clairement identifiables. Les douleurs sont présentées, mais non ressenties, ce qui les différencie des douleurs psychosomatiques et somatoformes.

\section{Somatisation}

Présentation de symptômes physiques sans cause organique décelable dans des situations de stress psychique (ancienne dénomination: trouble fonctionnel).

\section{Status quo ante}

Lorsqu'il existe une pathologie initiale (maladive) non imputable à l'accident, celle-ci peut être aggravée par l'accident. Si cette aggravation n'est que temporaire, on atteint à un moment donné le status quo ante, dans lequel l'état maladif antérieur est revenu au stade où il se trouvait avant l'accident [3].

\section{Status quo sine}

Si la pathologie initiale, qui existait avant l'accident, connaît de toute façon une évolution progressive, le status quo sine est atteint au moment où l'aggravation temporaire imputable à l'accident n'a plus d'incidence sur le déroulement inéluctable de la maladie [3].

\section{Taux d'invalidité}

Pour évaluer le taux d'invalidité, le revenu que l'assuré aurait pu obtenir s'il n'était pas invalide est comparé avec celui qu'il pourrait obtenir en exerçant l'activité qui peut raisonnablement être exigée de lui après les traitements et les mesures de réadaptation, sur un marché du travail équilibré (art. 16 LPGA)

\section{TFA}

Tribunal fédéral des assurances, Lucerne (à partir du $1^{\text {er }}$ janvier 2007: Cours de droit social du Tribunal fédéral, Lucerne).

\section{Références}

1 Meyer-Blaser U. Der Rechtsbegriff der Arbeitsunfähigkeit und seine Bedeutung in der Sozialversicherung, namentlich für den Einkommensvergleich in der Invalidenversicherung. In «Schmerz und Arbeitsunfähigkeit», Schriftenreihe IRP-HSG, Band 23, St. Gallen (2003), S. $27 \mathrm{ff}$.

2 Villiger P, Seitz M. Rheumatologie in Kürze. 2. Auflage. Stuttgart: Thieme Taschenbuch; 2006.

3 Meine J, Burri P. Leitfaden UVG für beratende Ärzte und Versicherungsfachleute. 2. Auflage. Zürich: Schweizerischer Versicherungsverband; 1998.

\section{Références ultérieures}

- Rompe G, Erlenkämper A. Begutachtung der Haltungs- und Bewegungsorgane. 4. Auflage. Stuttgart: Thieme; 2004.

- Oliveri M, et al. Grundsätze der ärztlichen Beurteilung der Zumutbarkeit und Arbeitsfähigkeit. Teil 1: Schweiz Med Forum. 2006;6:420-31; Teil 2: Schweiz Med Forum. 2006;6:448-54. 